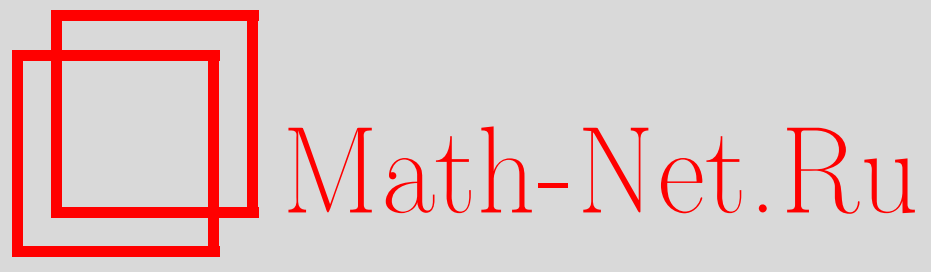

В. П. Гришухин, Свободные и несвободные многогранники Вороного, Матем. заметки, 2006, том 80, выпуск $3,367-378$

DOI: https://doi.org/10.4213/mzm2822

Использование Общероссийского математического портала Math-Net.Ru подразумевает, что вы прочитали и согласны с пользовательским соглашением http://www .mathnet.ru/rus/agreement

Параметры загрузки:

IP : 3.85 .7 .115

26 апреля 2023 г., 14:22:19

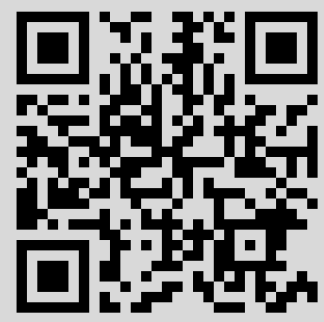




\title{
СВОБОДНЫЕ И НЕСВОБОДНЫЕ МНОГОГРАННИКИ ВОРОНОГО
}

\section{В. П. Гришухин}

\begin{abstract}
Многогранник Вороного некоторой точки $v$ трансляционной решетки есть замыкание множества точек пространства, более близких к $v$, чем к другим точкам решетки. Многогранник Вороного есть частный случай параллелоэдра, т.е. многогранника, параллельные сдвиги которого заполняют все пространство без зазоров и пересечений по внутренним точкам. Сумма Минковского параллелоэдра с отрезком не всегда есть параллелоэдр. Параллелоэдр $P$ называется свободным вдоль вектора $e$, если сумма $P$ с отрезком прямой, натянутой на $e$, есть параллелоэдр. Доказывается теорема о том, что если многогранник Вороного $P_{V}(f)$ квадратичной формы $f$ свободен вдоль некоторого вектора, то для любой формы $g$ из замыкания L-области формы $f$ ее многогранник Вороного $P_{V}(g)$ тоже свободен вдоль некоторого вектора. Для двойственной корневой решетки $E_{6}^{*}$ и для бесконечной серии решеток $D_{2 m}^{+}$, где $m \geqslant 4$, доказано, что их многогранники Вороного несвободны во всех направлениях.
\end{abstract}

Библиография: 14 названий.

1. Введение. В последнее время большое внимание уделяется исследованию разбиения пространства на многогранники, в частности, на параллелоэдры и многогранники Делоне. Параллелоэдр - это такой многогранник, параллельными сдвигами которого можно замостить все пространство без пустот и пересечений по внутренним точкам. Частным случаем параллелоэдра является многогранник Вороного некоторой точки $v$ решетки, состоящий из всех точек пространства, расположенных от $v$ не дальше, чем от других точек решетки.

Топологически двойственным к разбиению пространства на параллелоэдры Вороного является разбиение на многогранники Делоне. Многогранник Делоне, соответствующий некоторой вершине $w$ разбиения на параллелоэдры, есть выпуклая оболочка центров $v_{i}$ параллелоэдров $P_{i}$, имеющих $w$ в качестве вершины.

Матрица Грама базиса $n$-мерной решетки центров параллелоэдров разбиения пространства на многогранники Вороного является матрицей положительно определенной квадратичной формы от $n$ переменных. Все положительно определенные квадратичные формы от $n$ переменных образуют открытый конус в $N_{L}$-мерном пространстве всех $n \times n$ матриц, где $N_{L}=n(n+1) / 2$.

Г. Вороной определил L-тип параллелоэдра и его решетки. B современных терминах L-mun решетки есть класс изоморфизма частично упорядоченного по включению множества граней ее многогранника Вороного. Связное множество всех квадратичных форм, определяющих решетки одного L-типа, образует область L-типа, 
или L-областъ. Всякая L-область есть открытый многогранный подконус замкнутого конуса всех положительно полуопределенных квадратичных форм. L-область максимальной размерности $N_{L}$ называется общей. L-область размерности, меньшей чем $N_{L}$, называется специальной. Всякая специальная L-область размерности $k$ есть $k$-мерная грань замыкания некоторых общих L-областей. Так как всякая L-область открыта, то любая положительно определенная форма принадлежит ровно одной L-области.

В мало известной статье [1] Б. А. Венков ввел понятие многогранника ненулевой толщины в направлении некоторого $k$-мерного подпространства. Он изучал параллелоэдры ненулевой толщины. В частности, в теореме 2 статьи [1] он доказал, что $n$-мерный многогранник Вороного имеет ненулевую толщину вдоль $k$-мерного подпространства тогда и только тогда, когда ортогональное $(n-k)$-мерное подпространство заполнено многогранниками Делоне размерности не больше $n-k$. В этом случае, как показал Венков, область L-типа такого многогранника Вороного имеет $(k(k+1) / 2)$-мерную грань, состоящую из форм ранга $k$.

Эти результаты особенно существенны в случае $k=1$, когда $(n-k)$-мерное подпространство становится гиперплоскостью, разбитой на фасеты многогранников Делоне разбиения Делоне, а область L-типа имеет крайний луч, натянутый на форму ранга 1. Соответствующая гиперплоскость названа в [2] слоевой плоскостъю. Таким образом существует взаимно однозначное соответствие между

(а) многогранником Вороного ненулевой толщины вдоль прямой $\ell$,

(б) слоевой плоскостью, ортогональной прямой $\ell$,

(в) крайним лучом ранга 1 замыкания области L-типа этого многогранника Boроного.

Ненулевая толщина многогранника вдоль прямой $\ell$ эквивалентна существованию замкнутой зоны ребер, параллельных $\ell$. Замкнутые зоны множества параллельных между собой ребер параллелоэдра изучал П. Энгель в ряде своих работ, см., например, [3]. Реберная зона называется замкнутой, если каждая двумерная грань содержит либо два, либо ни одного ребра этой зоны. Энгель рассматривает сжатие параллелоэдра вдоль ребер замкнутой зоны до тех пор, пока эта зона не перестает быть замкнутой, т.е. становится открытой. Энгель называет параллелоэдр зонно сжатым, если все его зоны открыты, и максималъным, если он не может быть получен сжатием зон из другого параллелоэдра.

Если все крайние лучи замыкания некоторой L-области натянуты на формы ранга 1, то такая область названа в [4] дайсинг областью. Решетка, соответствующая форме дайсинг области, есть пересечение гиперплоскостей, каждая из которых в этом случае является слоевой плоскостью. В [4] ставится вопрос о существовании общих L-областей, замыкание которых не имеет крайних лучей ранга 1 . B частности там приводится пример такой L-области форм от $n=8$ переменных, один из крайних лучей которой натянут на форму унимодулярной корневой решетки $E_{8}$. Энгель с помощью компьютера показал, что этим свойством обладает и решетка меньшей размерности, а именно, решетка $E_{6}^{*}$, см. [3].

В этой статье я попытался более ясно доказать этот факт для $E_{6}^{*}$ и целой серии решеток $D_{2 m}^{+}$, включающей решетку $E_{8}=D_{8}^{+}$. Бесконечная серия решеток $D_{2 m}^{+}$ обладает рядом свойств, замечательных в некоторых отношениях. Метрическая форма решетки $D_{2 m}^{+}$для $m \geqslant 3$ лежит на крайнем луче разбиения на области L- 
типов конуса всех положительно полуопределенных квадратичных форм. Если $m$ кратно четырем, $m=4 k$, то $D_{8 k}^{+}$есть четная унимодулярная решетка.

В [5] показано, что всякий параллелоэдр ненулевой толщины вдоль прямой есть сумма Минковского некоторого параллелоэдра с отрезком этой прямой. Для многих решеток сумма Минковского их многогранника Вороного с отрезком прямой в некотором направлении е есть параллелоэдр. Я называю такой многогранник Вороного свободным вдоль (или в направлении) вектора $е$. В противном случае он называется несвободным вдоль е. Отмечу, что все многогранники Вороного размерности 5 свободны, по крайней мере, вдоль десяти направлений. Это значит, что замыкание всякой общей L-области положительно определенных форм от 5 переменных имеет не менее 10 крайних лучей ранга 1.

Разумеется, вектор е рассматривается лежащим в пространстве многогранника Вороного. Сумма любого параллелоэдра $P$ с отрезком прямой вдоль вектора $e$, не лежащего в пространстве $P$, всегда есть параллелоэдр, являющийся призмой с основанием $P$.

Очевидно, что понятия свободы и несвободы применимы и к параллелоэдру, не являющемуся многогранником Вороного. Замечу, что прибавление отрезка к параллелоэдру тесно связано с зонным растяжением параллелоэдра, изучаемым Энгелем в ряде работ, в частности, в [3]. Но он не дает условия возможности такого растяжения, а изучает ее экспериментально, т.е. с помощью компьютера. Согласно определению одновременно максимальный и зонно сжатый параллелоэдр не допускает растяжения. В моих терминах такой параллелоэдр несвободен во всех направлениgX.

Оказывается, что для $m \geqslant 4$ многогранник Вороного решетки $D_{2 m}^{+}$несвободен ни в каком направлении. Из доказываемой в этой статье основной теоремы, сформулированной в аннотации, следует, что для любой L-области $\mathscr{D}$, замыкание которой $\operatorname{cl} \mathscr{D}$ имеет $D_{2 m}^{+}$крайним лучем, это замыкание $\operatorname{cl} \mathscr{D}$ не имеет крайних лучей ранга 1 , а многогранник Вороного $P_{V}(f)$ любой формы $f \in \mathscr{D}$ несвободен ни в каком направлении.

2. Минимальные векторы классов смежности и области L-типов. Минимальные векторы классов смежности $L / 2 L$ важны для изучения строения разбиения пространства на многогранники Вороного и двойственного к нему разбиения на многогранники Делоне, а значит, и для определения L-типа решетки $L$.

Будем рассматривать вектор $n$-мерного пространства как вектор-столбец, т.е. как $(n \times 1)$-матрицу с одним столбцом. Обозначим через $A^{T}$ транспонированную матрицу $A$. Если $x$ есть вектор-столбец, то $x^{T}$ есть вектор-строка, и $x^{T} y$ есть скалярное произведение вектор-столбцов $x$ и $y$. Скалярный квадрат $x^{T} x$ назовем нормой вектора $x$.

Вместе с вектором $v$ его класс смежности содержит и вектор $-v$ той же нормы $v^{2}$. Вектор называется минималъным в своем классе смежности, если он имеет минимальную норму среди всех векторов своего класса смежности. Класс смежности называется простым, если он имеет единственный, с точностью до знака, минимальный вектор. Остальные классы называются непростыми.

Минимальные векторы простых классов смежности всякой решетки определяют фасеты многогранника Вороного этой решетки. Следуя Венкову [1], будем называть минимальные векторы простых классов смежности основными векторами. Каждый 
основной вектор соединяет центры двух многогранников Вороного, смежных по фасете. Множество минимальных векторов непростого класса смежности является множеством диагоналей центрально симметричных граней некоторых многогранников Делоне разбиения Делоне этой решетки.

L-тип решетки определяется также комбинаторным типом разбиения Делоне, т.е. разбиения на многогранники Делоне. С каждой точкой $v_{0}$ решетки связана звезда $\operatorname{St}\left(v_{0}\right)$ многогранников Делоне, имеющих точку $v_{0}$ вершиной. Каждая вершина $v$ многогранника Делоне звезды $\operatorname{St}\left(v_{0}\right)$ есть концевая точка решеточного вектора $v-v_{0}$, который является минимальным вектором в своем классе смежности. Каждый многогранник Делоне вписан в сферу.

Справедлив следующий факт (см., например, [6]), являющийся обобщением теоремы Пифагора. Пусть в $n$-мерном пространстве имеются $n+1$ векторов $v_{i}, 1 \leqslant$ $i \leqslant n+1$, таких, что между ними существует единственная линейная зависимость $\sum_{i=1}^{n+1} \lambda_{i} v_{i}=0, \lambda_{i} \in \mathbb{R}$. Тогда $n+1$ концевая точка этих векторов и начало 0 лежат на сфере тогда и только тогда, когда справедливо равенство $\sum_{i=1}^{n+1} \lambda_{i} v_{i}^{2}=0$, т.е. нормы этих векторов подчиняются той же зависимости, что и сами векторы.

В случае, когда все $v_{i}$ являются основными векторами решетки, выпуклая оболочка $n+2$ точек решетки, лежащих на сфере, есть многогранник Делоне, называемый многогранником переделивания общего вида. Он является многократной пирамидой над простым многогранником переделивания, порожденным теми векторами $v_{i}$, для которых $\lambda_{i} \neq 0$. Многогранники переделивания находятся во взаимно однозначном соответствии c L-областями, являющимися фасетами общих L-областей размерности $N_{L}=n(n+1) / 2$. (Подробности см., например, в [2] и [7]).

Введем для удобства множество $N=\{1,2, \ldots, n\}$. Пусть $v=\sum_{i \in N} z_{i}(v) b_{i}$ есть целочисленное представление вектора $v$ решетки $L(f)$ в ее базисе $B_{f}=\left\{b_{i}: i \in N\right\}$, соответствующем квадратичной форме $f(x)$. Тогда норма вектора $v=\sum_{i \in N} z_{i}(v) b_{i}$ равна $v^{T} v=f(z(v))=\sum_{i, j \in N} a_{i j} z_{i}(v) z_{j}(v)$. Она есть линейная комбинация коэффициентов $a_{i j}$ квадратичной формы $f$.

Поэтому, если многогранник Делоне $P_{D}$ решетки $L(f)$ отличен от симплекса, то имеются линейные зависимости между векторами, представляющими вершины многогранника $P_{D}$, и их нормами. Эти линейные зависимости определяют уравнения, описывающие зависимости между коэффициентами $a_{i j}$ формы $f$ решетки $L(f)$. Эти уравнения являются инвариантом L-области формы $f$. Если при изменении параметров $a_{i j}$ некоторая вершина уходит со сферы, описанной вокруг $P_{D}$, то соответствующее равенство превращается в неравенство.

Таким образом, звезда $\operatorname{St}(0)$ многогранников Делоне с вершиной $v_{0}=0$ определяет систему уравнений, неизвестными которой являются коэффициенты $a_{i j}$. Эта система уравнений описывает в $N_{L}$-мерном пространстве всех матриц $a_{i j}, i, j \in N$, подпространство, натянутое на L-область $\mathscr{D}(f)$ формы $f$. Поэтому размерность $\mathscr{D}(f)$ равна корангу соответствующей системы равенств. В [6] и [8] подробно описывается строение этой системы. Там же размерность L-области $\mathscr{D}(f)$ названа степенъю нежесткости решетки $L(f)$ и ее формы $f$ и показано, как вычислять степень нежесткости. Замечу, что из этой системы равенств, описывающей $\mathscr{D}(f)$, вытекает подсистема, получающаяся приравниванием норм минимальных векторов непростых классов смежности решетки $L(f)$. Будучи многогранным множеством, L-область $\mathscr{D}(f)$ определяется описанными выше равенствами и неравенствами. 
Итак, L-область есть многогранный конус, размерность которого лежит в пределах от 1 до $N_{L}$. L-области размерности 1 являются крайними лучами замыкания L-областей бо́льших размерностей. Решетка и форма L-области размерности 1 называются жесткими. Понятие жесткости решетки и ее разбиения Делоне впервые ввел Е. П. Барановский в [9].

Ранг матрицы коэффициентов $\left(a_{i j}\right)$ формы $f=\sum_{i, j \in N} a_{i j} x_{i} x_{j}$ называется рангом формы $f$. Очевидно, что формы одной L-области имеют одинаковый ранг. Назовем рангом L-области ранг ее форм. Замечу, что замыкание L-области может иметь грань, являющуюся множеством форм меньшего ранга. В [1] Венков показал, что если замыкание L-области содержит форму ранга $k$, то унимодулярным преобразованием ее можно превратить в положительную форму от $k$ переменных. Это значит, что любой форме $g$ из замыкания L-области соответствует многогранник Вороного $P_{V}(g)$, размерность которого есть ранг формы $g$. Особую роль играют одномерные L-области ранга 1 , т.е. крайние лучи ранга 1.

3. Свободные многогранники Вороного. В [1] Венков ввел понятие параллелоэдра ненулевой толщины вдоль некоторого $k$-мерного подпространства. Наиболее интересными оказываются параллелоэдры ненулевой толщины вдоль прямой.

Ниже приведенная теорема, доказанная в [10], несколько обобщает результат Венкова [1] для $k=1$, упомянутый во введении.

TeOpema 1. Пусть $\mathscr{D}(f)$ есть L-область квадратичной формы $f$ и ее решетки $L(f)$. Пусть $B_{f}=\left\{b_{i}: i \in N\right\}$ есть базис решетки $L(f)$, определяемый формой $f$. Пусть $Н$ есть гиперплоскость, натянутая на $(n-1)$-мерную подрешетку в решетке $L(f)$, и е есть единичный вектор, ортогональный $Н$. Следующие утверждения эквивалентны:

1)

2) многогранник Вороного $P_{V}(f)$ имеет замкнутую зону ребер, параллельных е;

3) многогранник Вороного $P_{V}(f)$ имеет ненулевую толщину вдоль е;

4) форма $f_{e}(x)=\left(e^{T} \sum_{i \in N} b_{i} x_{i}\right)^{2}$ ранга 1 лежит на крайнем луче замыкания L-области $\mathscr{D}(f)$.

В [5] показано, что всякий параллелоэдр ненулевой толщины вдоль прямой есть сумма Минковского некоторого параллелоэдра с отрезком этой прямой. Оказывается, что сумма параллелоэдра с отрезком не всегда является параллелоэдром. Скажем, что параллелоэдр $P$ свободен вдоль (или в направлении) вектора е, если его сумма Минковского $P+Q_{e}$ с отрезком $Q_{e}$ прямой, натянутой на вектор $e$, есть параллелоэдр. В частности, параллелоэдр ненулевой толщины вдоль $e$ всегда свободен вдоль $e$. Если $P+Q_{e}$ не есть параллелоэдр, то $P$ называется несвободным вдоль $е$.

Напомню, что если вектор $е$ не лежит в пространстве, натянутом на параллелоэдр $P$, то $P+Q_{e}$ всегда есть параллелоэдр, являющийся призмой с основанием $P$. Поэтому $P$ свободен вдоль любого вектора, не лежащего в пространстве $P$. Ниже, говоря о свободе или несвободе параллелоэдра $P$ вдоль вектора $e$, подразумевается, что $e$ лежит в линейной оболочке Aff $P$, если не утверждается иное.

Замечу, что ортогональная вектору $е$ гиперплоскость $H$, упомянутая в теореме 1 , не является слоевой плоскостью, если $P_{V}(f)$ свободен, но имеет нулевую толщину 
вдоль $e$. Но все же в этом случае $H$ обладает некоторыми свойствами слоевой плоскости. Например, она может пересекать по внутренним точкам только центрально симметричные многогранники Делоне и только так, что она проходит через их центры симметрии.

В [11] Венков доказал, что $n$-мерный многогранник $P$ является параллелоэдром тогда и только тогда, когда он и все его фасеты центрально симметричны, а его проекция вдоль любой $(n-2)$-мерной грани есть либо параллелограмм, либо центрально симметричный шестиугольник.

Фасеты, проектируемые в ребра параллелограмма или шестиугольника, образуют 4- u 6-поясок, соответственно. Вектор, ортогональный фасете некоторого многогранника, называется фасетным вектором этого многогранника. Фасетные векторы пояска параллелоэдра лежат в двумерной плоскости, ортогональной $(n-2)$ грани этого параллелоэдра, вдоль которой проектируются пояски. Таким образом, 6 -поясок определяется тремя линейно зависимыми фасетными векторами $p_{i}, p_{j}$ и $p_{k}$. Если $F_{i}, F_{j}$ и $F_{k}$ есть три фасеты 6 -пояска, идущие подряд в этом порядке, то всегда можно подобрать длины фасетных векторов так, чтобы выполнялось равенство $p_{i}+p_{k}=p_{j}$. Если $P$ есть многогранник Вороного, то фасетные векторы совпадают с основными векторами $v_{i}, v_{j}$ и $v_{k}$, а равенство $v_{i}+v_{k}=v_{j}$ для основных векторов выполняется автоматически, так как эти векторы порождают двумерную решетку.

В [5] доказано следующее утверждение.

ПреДЛОЖЕНИЕ 1. Для параллелоэдра Р и вектора е следующие утверждения эквивалентны:

1) Р свободен вдоль е;

2) $e^{T} p=0$ хотя бы для одного фасетного вектора $p$ из тройки фасетных векторов каждого 6-пояска в $P$.

В качестве иллюстрации предложения 1 найдем все направления, вдоль которых свободен многогранник Вороного корневой решетки $D_{n}$ (т.е. $n$-мерной решетки второй совершенной формы), тем более, что этот факт понадобится в дальнейшем.

Пусть $\left\{e_{i}: 1 \leqslant i \leqslant n\right\}$ есть ортонормированный базис пространства $\mathbb{R}^{n}$. Поэтому $e_{i}^{T} e_{i}=1,1 \leqslant i \leqslant n, e_{i}^{T} e_{j}=0,1 \leqslant i<j \leqslant n$. Пусть $N=\{1,2, \ldots, n\}$. Для любого подмножества $S \subseteq N$ положим $\bar{S}=N-S$, и $e(S)=\sum_{i \in S} e_{i}$. Обозначим через $|S|$ мощность множества $S$.

ПреДЛОЖЕНИЕ 2. Многогранник Вороного $P_{V}\left(D_{n}\right)$ корневой решетки $D_{n}$ nри $n \geqslant 3$ свободен вдоль $n+2^{n-1}$ направлений, заданных векторами $e_{i}, i \in N, u$ $e(S)-e(\bar{S})$ для всех $S \subseteq N$.

ДокаЗАтельство. Вектор $x=\sum_{i \in N} x_{i} e_{i}$ есть вектор решетки $D_{n}$, если $x_{i}$ все целые и $\sum_{i \in N} x_{i} \equiv 0(\bmod 2)$. Хорошо известно, что фасетные векторы $P_{V}\left(D_{n}\right)$ есть корни $e_{i}-e_{j}, e_{i}+e_{j}, i, j \in N, i \neq j$. Согласно предложению 1 нужно найти такие векторы $z=\sum_{i \in N} z_{i} e_{i}$, что $p^{T} z=0$ хотя бы для одного вектора $p$ из тройки фасетных векторов каждого 6-пояска.

Существуют следующие 2 типа троек фасетных векторов, определяющих 6-пояски решетки $D_{n}$,

a) $e_{i}-e_{j}, e_{j}-e_{k}, e_{i}-e_{k}, 1 \leqslant i<j<k \leqslant n$;

б) $e_{i}+e_{j}, e_{j}+e_{k}, e_{i}-e_{k}, 1 \leqslant i<j<k \leqslant n$; 
Вектор $z$ не может иметь три попарно неравных координаты. Если такие три координаты существуют, скажем $z_{i}, z_{j}, z_{k}$, то $z$ не ортогонален ни одному вектору тройки $e_{i}-e_{j}, e_{j}-e_{k}, e_{i}-e_{k}$ вида а). Поэтому вектор $z$ должен иметь вид $z=$ $z^{\prime}(S)=z_{1} e(S)+z_{2} e(\bar{S})$, где $z_{1} \neq z_{2}$ и $S \subseteq N$. Легко видеть, что хотя бы один вектор из каждой тройки вида а) ортогонален $z^{\prime}(S)$, так как хотя бы одна пара индексов из тройки индексов $\{i, j, k\}$ лежит либо в $S$, либо в $\bar{S}$.

Для того, чтобы $z^{\prime}(S)$ был ортогонален хотя бы одному вектору из каждой тройки $e_{i}+e_{j}, e_{j}+e_{k}, e_{i}-e_{k}$ вида б), нужно, чтобы было либо $S=N$, либо $\bar{S}=N$, либо $z_{2}=0$ и $|S|=1$, либо $z_{1}=0$ и $|\bar{S}|=1$, либо $z_{1}+z_{2}=0$. Последнее условие необходимо для того, чтобы $z^{\prime}(S)$ был ортогонален таким тройкам, что $\{i, j\} \subseteq S$, $k \in \bar{S}$ или $k \in S,\{i, j\} \subseteq \bar{S}$ (напомню, что $z_{1} \neq z_{2}$ ). Поэтому вектор $z$ с точностью до множителя должен иметь либо вид $z(S)=e(S)-e(\bar{S}), S \subseteq N$, либо $z=e_{i}, i \in N$.

4. L-области свободных и несвободных многогранников Вороного. Напомню, что всякая L-область $\mathscr{D}$ есть открытый многогранный конус размерности $k$, $1 \leqslant k \leqslant N_{L}$. Любая квадратичная форма $f \in \mathscr{D}$ ранга $n$ определяет базис $B_{f}=$ $\left\{b_{i}: i \in N\right\}$ своей решетки $L(f)$. Будем понимать $B_{f}$ как $n \times n$ матрицу, столбцами которой являются базисные векторы $b_{i}, i \in N$. Каждый решеточный вектор $v \in L(f)$ имеет в базисе $B_{f}$ однозначное целочисленное представление $v=\sum_{i \in N} z_{i}(v) b_{i}$, $z_{i}(v) \in \mathbb{Z}$. Запишем это представление в матричном виде $v=B_{f} z(v)$, где $z(v)=$ $\left(z_{i}(v): i \in N\right)$ есть целочисленный вектор-столбец, представляющий $v \in L(f)$. Тройке $\left(v_{i}, v_{j}, v_{k}\right)$ основных векторов каждого 6-пояска, где $v_{i}+v_{k}=v_{j}$, соответствует тройка $\left(z\left(v_{i}\right), z\left(v_{j}\right), z\left(v_{k}\right)\right)$ целочисленных векторов, удовлетворяющих тому же равенству $z\left(v_{i}\right)+z\left(v_{k}\right)=z\left(v_{j}\right)$.

Если $f$ имеет ранг $n$, то $L(f)=B_{f} \mathbb{Z}^{n}$, где $\mathbb{Z}^{n}$ есть решетка всех $n$-мерных целочисленных векторов. Рассмотрим вектор $e_{0}$ и ортогональную ему подрешетку в $\mathbb{Z}^{n}$ :

$$
\mathbb{Z}_{0}=\left\{z \in \mathbb{Z}^{n}: e_{0}^{T} z=0\right\} .
$$

Подрешетка $\mathbb{Z}_{0}$ порождает в $L(f)$ подрешетку

$$
L_{0}(f)=\left\{v \in L(f): v=B_{f} z, z \in \mathbb{Z}_{0}\right\} .
$$

Подрешетка $L_{0}(f)$ состоит из решеточных векторов $v$, ортогональных вектору $e_{f}=$ $\left(B_{f}^{-1}\right)^{T} e_{0}$. Действительно, $e_{f}^{T} v=e_{0}^{T} B_{f}^{-1} B_{f} z=e_{0}^{T} z=0$. Поэтому $L_{0}(f)$ лежит в гиперплоскости $H_{f}=\left\{x \in \mathbb{R}^{n}: e_{f}^{T} x=0\right\}$, ортогональной вектору $e_{f}$.

Согласно предложению 1 многогранник Вороного $P_{V}(f)$ свободен вдоль вектора $e_{f}$ тогда и только тогда, когда подрешетка $L_{0}(f)$ содержит хотя бы один вектор тройки основных векторов каждого 6-пояска. Это значит, что подрешетка $\mathbb{Z}_{0}$ содержит целочисленные вектора, представляющие хотя бы один вектор тройки каждого 6-пояска.

Моя цель - показать, что если $P_{V}(f)$ свободен вдоль $e_{f}$, то многогранник Вороного $P_{V}(g)$ свободен вдоль $e_{g}=\left(B_{g}^{-1}\right)^{T} e_{0}$ для любой формы $g$ любой L-области, являющейся гранью замыкания L-области $\mathscr{D}$ формы $f$.

Пусть $\mathscr{Z}(f)$ есть множество целочисленных представлений основных векторов $v$ решетки $L(f)$. Целочисленные векторы $z(v) \in \mathscr{Z}(f)$ не сравнимы между собой по модулю 2. В этом случае говорят, что они принадлежат различным классам четности. Множество $\mathscr{Z}(f)$ является инвариантом L-области $\mathscr{D}$, т.е. $\mathscr{Z}(f)=\mathscr{Z}(\mathscr{D})$ не 
зависит от $f$ для $f \in \mathscr{D}$. Заметим, что множество $\mathscr{Z}(\mathscr{D})$ не является полным инвариантом L-области $\mathscr{D}$, т.е. оно не определяет $\mathscr{D}$ однозначно. Например, $\mathscr{Z}(\mathscr{D})$ одно и тоже для общей области $\mathscr{D}$ и тех фасет ее замыкания, которые соответствуют многогранникам переделивания, отличным от пирамид над квадратом. Согласно определению области С-типа в [7] множество $\mathscr{Z}(\mathscr{D})$ есть полный инвариант С-области, содержащей $\mathscr{D}$.

ПреДЛОЖЕнИЕ 3. Пусть $\mathscr{D}^{\prime}$ есть некоторая гранъ замыкания L-области $\mathscr{D}$, обозначаемого через $\mathrm{cl} \mathscr{D}, \mathscr{D}^{\prime} \subset \operatorname{cl} \mathscr{D}$, и ранг $\mathscr{D}^{\prime}$ равен рангу $\mathscr{D}$. Тогда $\mathscr{Z}\left(\mathscr{D}^{\prime}\right) \subseteq \mathscr{Z}(\mathscr{D})$ и множество троек целочисленных векторов 6-поясков, содержащихся в $\mathscr{Z}\left(\mathscr{D}^{\prime}\right)$, является подмножеством троек, содержащихся в $\mathscr{Z}(\mathscr{D})$.

ДокАзАтЕЛЬСтво. Напомню, что L-область $\mathscr{D}$ определяется равенствами и неравенствами, наложенными на линейные зависимости между нормами минимальных векторов классов смежности решетки $L(f)$ для $f \in \mathscr{D}$. Когда форма $f$ передвигается в некоторую L-область, являющуюся гранью $\mathscr{D}^{\prime}$ области $\mathscr{D}$ того же ранга, то появляются новые зависимости между нормами минимальных векторов, в частности, некоторые классы смежности из простых классов превращаются в непростые. В результате множество основных векторов не расширяется, но чаще всего даже сокращается. Отсюда вытекает первая часть доказываемого утверждения.

Так как ранг $\mathscr{D}^{\prime}$ равен рангу $\mathscr{D}$, то новые тройки зависимых минимальных векторов возникнуть не могут. В противном случае трехмерная подрешетка, порожденная этими тремя векторами, вырождается в двумерную, и ранг L-области понижается. Это доказывает вторую часть утверждения предложения.

ПреДЛОЖЕНИЕ 4. Пусть многогранник Вороного $P_{V}(f)$ формы $f \in \mathscr{D}$ свободен вдоль вектора е. Тогда для любой формы $g \in \mathrm{cl} \mathscr{D}$ того же ранга, что $u f$, многогранник Вороного $P_{V}(g)$ свободен вдоль вектора $e_{g}=\left(B_{g}^{-1}\right)^{T} B_{f}^{T}$ e.

ДоказАТЕЛЬСтво. Положим $e=e_{f}$ и $e_{0}=B_{f}^{T} e_{f}$. Вектор $e_{0}$ определяет ортогональную ему подрешетку $\mathbb{Z}_{0} \subseteq \mathbb{Z}^{n}$. Так как $P_{V}(f)$ свободен вдоль $e_{f}$, то в пересечении $\mathscr{Z}(\mathscr{D}) \cap \mathbb{Z}_{0}$ содержатся целочисленные векторы, представляющие некоторые векторы троек каждого 6-пояска $P_{V}(f)$. Так как согласно предложению 3 $\mathscr{Z}\left(\mathscr{D}^{\prime}\right) \subseteq \mathscr{Z}(\mathscr{D})$ и множество представлений троек $\mathscr{D}^{\prime}$ содержится в множестве представлений троек области $\mathscr{D}$, то $\mathscr{Z}\left(\mathscr{D}^{\prime}\right) \cap \mathbb{Z}_{0}$ содержит хотя бы один вектор, представляющий вектор тройки каждого 6-пояска $P_{V}(g)$ для любой формы $g \in \mathscr{D}^{\prime}$. Если $z$ есть такой вектор, то основной вектор $B_{g} z$ ортогонален вектору $e_{g}=\left(B_{g}^{-1}\right)^{T} e_{0}$. Поэтому для $P_{V}(g)$ выполнено условие 2) предложения 1. Следовательно, $P_{V}(g)$ свободен вдоль вектора $e_{g}=\left(B_{g}^{-1}\right)^{T} e_{0}=\left(B_{g}^{-1}\right)^{T} B_{f}^{T} e$.

Теорема 2. Пусть многогранник Вороного $P_{V}(f)$ формы $f \in \mathscr{D}$ свободен в некотором направлении. Тогда для любой формы $g \in \mathrm{cl} \mathscr{D}$ многогранник Вороного $P_{V}(g)$ свободен вдоль некоторого вектора пространства, натянутого на $P_{V}(f)$.

ДокАзАтЕЛьство. Если ранг $g$ равен рангу $f$, то утверждение теоремы следует из предложения 4. Если ранг $g$ меньше ранга $f$, то многогранник Вороного $P_{V}(g)$ свободен вдоль любого вектора, лежащего в пространстве $P_{V}(f)$, но не принадлежащего пространству $P_{V}(g)$.

СлеДСТВИЕ 1. Пусть многогранник Вороного $P_{V}(g)$ формы $g \in \mathscr{D}_{0}$ несвободен ни в каком направлении. Тогда для любой формы $f$ из любой L-области $\mathscr{D}$, замъкание 
которой содержит L-область $\mathscr{D}_{0}$ в качестве грани, многогранник Вороного $P_{V}(f)$ несвободен ни в каком направлении.

ДокАЗАтЕЛЬСтво. Если существует вектор $e$, вдоль которого $P_{V}(f)$ свободен, то по теореме 2 многогранник $P_{V}(g)$ тоже свободен вдоль некоторого вектора. Полученное противоречие доказывает утверждение следствия.

Напомню, что всякий параллелоэдр, имеющий ненулевую толщину вдоль некоторого вектора, является свободным вдоль этого вектора. Поэтому эквивалентность 3$) \Longleftrightarrow 4$ ) теоремы 1 позволяет переформулировать следствие 1 в следующем виде.

СЛЕДСТвиЕ 2. Пусть многогранник Вороного $P_{V}(g)$ формы $g \in \mathscr{D}_{0}$ не свободен ни в каком направлении. Тогда замыкание всякой L-области, имеющее $\mathscr{D}_{0}$ в качестве грани, не имеет крайних лучей ранга 1.

Следствие 2 есть предложение 5, анонсированное в [5], но не доказанное там.

В следующем разделе будет доказана несвобода во всех направлениях решетки $E_{6}^{*}$ и бесконечной серии решеток $D_{2 m}^{+}$для $m \geqslant 4$. Для формы $g$ решетки $E_{8}=D_{8}^{+}$ результат следствия 2 упомянут в работе [4], но данная там его аргументация мне кажется недостаточной.

5. Несвобода решеток $E_{6}^{*}$ и $D_{2 m}^{+}$для $m \geqslant 4$. Очевидно, что три основных вектора, определяющих фасеты 6-пояска, образуют треугольник. Вообще, треугольник образуют любые три основных вектора, дающих с подходящими знаками в сумме нуль. Ребра этого треугольника являются ребрами разбиения Делоне. Этот треугольник может быть или не быть двумерной гранью некоторого многогранника Делоне. Напомню, что каждая $k$-мерная грань разбиения Делоне ортогональна некоторой $(n-k)$-мерной грани разбиения Вороного. Поэтому треугольная двумерная грань разбиения Делоне ортогональна $(n-2)$-мерной грани, в которой сходятся три фасеты разбиения Вороного. Следовательно, треугольник основных векторов соответствует 6-пояску тогда и только тогда, когда он есть двумерная грань разбиения Делоне.

П. Энгель с помощью компьютера показал, что многогранник Вороного $P_{V}\left(E_{6}^{*}\right)$ решетки $E_{6}^{*}$ является одновременно максимальным и зонно сжатым (см., например, [3; с. 28]). Это значит, что $P_{V}\left(E_{6}^{*}\right)$ несвободен во всех направлениях. Ниже этот факт доказывается аналитически с помощью предложения 1.

Я использую удобное описание решетки $E_{6}^{*}$, данное Е.П. Барановским в [12]. Пусть $e_{i}, 1 \leqslant i \leqslant 6$, есть базис такой, что $e_{i}^{T} e_{i}=2, e_{i}^{T} e_{j}=1 / 2,1 \leqslant i<j \leqslant 6$. Решетка $E_{6}^{*}$ есть центрировка векторами $h=\frac{1}{3} \sum_{i=1}^{6} e_{i}$ и $2 h$ решетки с базисом $\left\{e_{i}: 1 \leqslant i \leqslant 6\right\}$. Все классы смежности $E_{6}^{*}$ просты. Их минимальные векторы имеют нормы 2 и 3 , а именно 27 минимальных векторов $e_{i}, e_{i}-h, e_{i}+e_{j}-h, 1 \leqslant i<j \leqslant 6$, нормы 2 и 36 векторов $h, e_{i}-e_{j}, e_{i}+e_{j}+e_{k}-h, 1 \leqslant i<j<k \leqslant 6$, нормы 3.

Все многогранники Делоне конгруэнтны между собой и являются многогранниками типа переделивания $V_{222}^{6}$. Каждый из них является выпуклой оболочкой вершин трех правильных треугольников со сторонами нормы 3, расположенных в трех взаимно ортогональных плоскостях, пересекающихся в центре многогранника. Диаграмма ребер многогранника $V_{222}^{6}$ приведена на рис. 4 в [12]. Ребра $V_{222}^{6}$ имеют нормы 2 и 3 , и они нарисованы простыми и двойными линиями, соответственно. Из 
рисунка видно, что существуют треугольники основных векторов со следующими сочетаниями норм: $(3,3,3),(3,2,2)$ и $(2,2,2)$. При этом только треугольники типов $(3,2,2)$ и $(2,2,2)$ являются гранями $V_{222}^{6}$.

ПреДЛОЖеНИЕ 5. Многогранник Вороного $P_{V}\left(E_{6}^{*}\right)$ несвободен во всех направлениях.

ДокАЗАТЕЛЬСтво. Используем двойственный базис $e_{i}^{*}, 1 \leqslant i \leqslant 6$, такой, что $e_{i}^{T} e_{i}^{*}=1, e_{i}^{T} e_{j}^{*}=0,1 \leqslant i<j \leqslant 6$. Согласно предложению 1 будем искать вектор $z=\sum_{i=1}^{6} z_{i} e_{i}^{*}$ такой, что $p^{T} z=0$ хотя бы для одного вектора тройки фасетных векторов каждого 6-пояска.

Покажем, что все ненулевые координаты $z_{i} \neq 0$ одинаковы по величине. Предположим противное, и пусть $0 \neq z_{i} \neq z_{j} \neq 0$. Тогда для тройки $e_{i}-e_{j}, e_{i}, e_{j}$ типа $(3,2,2)$ все скалярные произведения $z^{T}\left(e_{i}-e_{j}\right)=z_{i}-z_{j}, z^{T} e_{i}=z_{i}, z^{T} e_{j}=z_{j}$ не равны нулю. Поэтому, с точностью до множителя, вектор $z$ имеет вид $\sum_{i \in S} e_{i}^{*}$, где $\varnothing \neq S \subseteq\{1,2,3,4,5,6\}$. Рассмотрим тройку $h, e_{i}, e_{i}-h$ типа $(3,2,2)$. Для $z=\sum_{i \in S} e_{i}^{*}$, где $S \ni i$, имеем $z^{T} h=\frac{1}{3}|S|, z^{T} e_{i}=1, z^{T}\left(e_{i}-h\right)=1-\frac{1}{3}|S|$. Поэтому для того, чтобы этот ненулевой вектор был ортогонален хотя бы одному вектору этой тройки, должно быть $|S|=3$. Итак, пусть $S=\{i, j, k\}$. Рассмотрим тройку $e_{i}+e_{j}+e_{k}-h, e_{j}+e_{k}-h, e_{i}$ типа $(3,2,2)$. Тогда $z^{T}\left(e_{i}+e_{j}+e_{k}-h\right)=2, z^{T}\left(e_{j}+e_{k}-h\right)=1$ и $z^{T} e_{i}=1$. Поэтому искомый вектор не существует, и по предложению 1 многогранник Вороного $P_{V}\left(E_{6}^{*}\right)$ не имеет свободных направлений.

Замечу, что в [6] доказано, что форма решетки $E_{6}^{*}$ жесткая.

Серия решеток $D_{2 m}^{+}$, где $m \geqslant 2$ есть целое число, обладает рядом интересных свойств. Она изометрична своей двойственной. Если $m$ кратно 4 , т.е. если размерность $n$ решетки $D_{n}^{+}$кратна 8 , то она есть четная унимодулярная решетка. Единственная четная унимодулярная решетка $E_{8}$ размерности 8 есть член этой серии для $m=4$. Решетка $D_{16}^{+}$есть одна из двух четных унимодулярных решеток размерности 16. Вторая решетка есть прямая сумма $E_{8} \oplus E_{8}$. Используя технику работ [8] и [6], нетрудно показать, что при $m \geqslant 3$ решетка $D_{2 m}^{+}$жесткая, т.е. ее L-область одномерна.

Определение решетки $D_{2 m}^{+}$дано в [13; раздел 7.3]. Эта решетка есть центрировка корневой решетки $D_{2 m}$ вектором, определяющим центр того ее многогранника Делоне, который есть полукуб.

Решетка $D_{n}^{+}$определена лишь для четных $n=2 m$. Вектор $x=\sum_{i \in N} x_{i} e_{i}$ есть вектор решетки, если

1) либо $x_{i}$ все целые и $\sum_{i \in N} x_{i} \equiv 0(\bmod 2)$,

2) либо $x_{i}$ все полуцелые и $\sum_{i \in N} x_{i} \equiv m(\bmod 2)$.

Минимальные векторы простых классов смежности, т.е. фасетные векторы, решетки $D_{2 m}^{+}$есть следующие векторы:

$$
e_{i}-e_{j}, \quad e_{i}+e_{j}, \quad i, j \in N, \quad i \neq j, \quad \frac{1}{2} \sum_{i \in N} \varepsilon_{i} e_{i}, \quad \varepsilon_{i} \in\{ \pm 1\}, \quad \Pi_{i \in N} \varepsilon_{i}=1 .
$$

Вектор третьего типа полезно записать в виде $\frac{1}{2}(e(S)-e(\bar{S}))$, где $S=\left\{i: i \in N, \varepsilon_{i}=\right.$ 1\} и $|S|$ четно.

Пример решетки $E_{6}^{*}$, двойственной к корневой решетке $E_{6}$, показывает, что существуют треугольники основных векторов, не соответствующие 6-пояскам. 
Лемма 1. Следующие 3 типа троек фасетных векторов определяют 6-пояски решетки $D_{2 m}^{+}$:

a) $e_{i}-e_{j}, e_{j}-e_{k}, e_{i}-e_{k}, 1 \leqslant i<j<k \leqslant n$;

б) $e_{i}+e_{j}, e_{j}+e_{k}, e_{i}-e_{k}, 1 \leqslant i<j<k \leqslant n$;

в) $\frac{1}{2} \sum_{i \in N} \varepsilon_{i} e_{i}, \varepsilon_{j} e_{j}+\varepsilon_{k} e_{k}, \frac{1}{2} \sum_{i \in N} \varepsilon_{i} e_{i}-\left(\varepsilon_{j} e_{j}+\varepsilon_{k} e_{k}\right), 1 \leqslant j<k \leqslant n$.

ДокАЗАтельство. Многогранник Вороного $P_{V}\left(D_{2 m}^{+}\right)$имеет в качестве вершин концевые точки векторов $e_{i}, i \in N$, и $\frac{1}{4} \sum_{i \in N} \varepsilon_{i} e_{i}-\varepsilon_{k} e_{k}, k \in N$. Эти вершины являются центрами многогранников Делоне типа ортаэдра (иначе, кросс-политопа). Грани всех размерностей ортаэдра являются симплексами. Легко убедиться, что двумерные грани ортаэдра с центром $e_{i}$ соответствуют тройкам типа а) и б). Аналогично, двумерные грани многогранника Делоне с центром $\frac{1}{4} \sum_{i \in N} \varepsilon_{i} e_{i}-\varepsilon_{k} e_{k}$ соответствуют тройкам типа в). Поэтому тройки а), б) и в) определяют 6 -пояски.

ПреДЛОЖЕНИЕ 6. Если $m \geqslant 4$, то многогранник Вороного решетки $D_{2 m}^{+}$не является свободным ни в каком направлении в его пространстве.

ДокАЗАТЕльство. Согласно предложению 1 нужно показать, что не существует такой вектор $z=\sum_{i \in N} z_{i} e_{i}$, что $p^{T} z=0$ хотя бы для одного вектора $p$ из каждой тройки фасетных векторов, приведенных в лемме 1. Тройки типа а) и б) это тройки решетки $D_{n}$. В предложении 2 было доказано, что вектор $z$ такой, что $p^{T} z=0$ хотя бы для одного вектора $p$ из каждой тройки фасетных векторов типа а) и б), имеет с точностью до множителя либо вид $z=z(S)=e(S)-e(\bar{S}), S \subseteq N$, либо $z=e_{i}$, $i \in N$.

Рассмотрим теперь тройки вида в). Легко видеть, что вектор $z$ вида $e_{i}$ для $i \in$ $\{j, k\}$ не ортогонален векторам тройки вида в). Пусть $z=z(S)$ и мощность $|S|$ четна. Не теряя общности, можно считать, что $|S| \geqslant 2$, иначе рассмотреть вектор $z(\bar{S})=-z(S)$ (напомню, что $n=2 m \geqslant 8)$. Тогда ни один вектор тройки вида

$$
\frac{1}{2}(e(S)-e(\bar{S})), \quad e_{j}+e_{k}, \quad \frac{1}{2}\left(e(S)-e(\bar{S})-\left(e_{j}+e_{k}\right)\right),
$$

где $j, k \in S$, не ортогонален $z(S)$. Если $|S|$ нечетно, то $S \neq N$ и существует $l \in N-S$. Не теряя общности, считаем, что $|S| \geqslant 3$. Рассмотрим тройки вида

$$
\frac{1}{2}\left(e(S)+e_{l}-e(\bar{S}-\{l\})\right), \quad e_{j}+e_{k}, \quad \frac{1}{2}\left(e(S)+e_{l}-e(\bar{S}-\{l\})\right)-\left(e_{j}+e_{k}\right),
$$

где $j, k \in S$. Так как $l \in \bar{S}$, имеем

$$
\begin{gathered}
z^{T}(S) \frac{1}{2}\left(e(S)+e_{l}-\left(e(\bar{S})-e_{l}\right)\right)=\frac{1}{2}\left(e^{2}(S)+e^{2}(\bar{S})-2\right)=\frac{1}{2}(n-2), \\
z^{T}(S)\left(e_{j}+e_{k}\right)=2 \\
z^{T}(S)\left(\frac{1}{2}\left(e(S)-e(\bar{S})+2 e_{l}\right)-\left(e_{j}+e_{k}\right)\right)=\frac{1}{2}(n-2)-2=\frac{1}{2}(n-6) .
\end{gathered}
$$

Поэтому, если $n>6$, то все три скалярных произведения не равны нулю. Итак, искомый вектор не существует.

Заметим, что при $n=6$ для вектора $z(S)=e(S)-e(\bar{S})$, где $|S|=|\bar{S}|=3$, в каждой тройке вида в) всегда существует вектор $p$ такой, что $z^{T}(S) p=0$. Это значит, что 
многогранник Вороного решетки $D_{6}^{+}$свободен вдоль этого вектора. Решетка $D_{6}^{+}$ обладает рядом замечательных свойств. Она есть решетка разрезов $\mathscr{L}_{4}$ в полном графе на четырех вершинах. Ее разбиение Делоне изучено в работе [14]. Кроме того, $\sqrt{2} D_{6}^{+}$есть слоистая решетка $\Lambda_{6}(3)$ с минимальной нормой 3 , упомянутая в [13] (см. с. 228 русского перевода). Напомним, что решетка $D_{6}^{+}$жесткая, т.е. ее L-область одномерна.

Предложения 5 и 6 и следствие 2 влекут следующее утверждение.

ПрЕДЛОЖЕНИЕ 7. Замыкание всякой L-области, имеющее крайний луч, натянутый на форму $E_{6}^{*}$ или $D_{2 m}^{+}$, где $m \geqslant 4$, не имеет крайних лучей ранга 1.

Итак, решетка $E_{6}^{*}$ есть минимальной размерности решетка, чей многогранник Вороного несвободен во всех направлениях. Из следствия 1 вытекает, что многогранник Вороного любой примитивной решетки, замыкание L-области которой имеет $E_{6}^{*}$ в качестве крайнего луча, тоже несвободен во всех направлениях. В размерности 5 и ниже таких решеток нет.

\section{СПИСОК ЦИТИРОВАННОЙ ЛИТЕРАТУРЫ}

[1] Б. А. Венков, “О проектировании параллелоэдров", Матем. сб., 49 (1959), 207-224.

[2] С. С. Рышков, Е. П. Барановский, "С-типы $n$-мерных решеток и пятимерные примитивные параллелоэдры (с приложением к теории покрытий)”, Тр. МИАН, 137 (1976).

[3] P. Engel, "Investigations of parallelohedra in $\mathbb{R}^{d ", ~ V o r o n o i ' s ~ I m p a c t ~ o n ~ M o d e r n ~ S c i e n c e, ~}$ 2, eds. P. Engel, H. Syta, Institute of Math., Kyiv, 1998, 22-60.

[4] R. Erdahl, S. S. Ryshkov, "On lattice dicing", European J. Combin., 15 (1994), 459-481.

[5] В. П. Гришухин, "Параллелоэдры ненулевой толщины”, Матем. сб., 195:5 (2004), 5978.

[6] E. P. Baranovskii, V.P. Grishukhin, "Non-rigidity degree of a lattice and rigid lattices", European J. Combin., 22 (2001), 921-935.

[7] S. S. Ryshkov, E. P. Baranovskii, "Repartitioning complexes in $n$-dimensional lattices (with full description for $n \leqslant 6$ )", Voronoi's Impact on Modern Science, 2, eds. P. Engel, H. Syta, Institute of Math., Kyiv, 1998, 115-124.

[8] Е. П. Барановский, В. П. Гришухин, "Алгоритм вычисления степени нежесткости Lразбиения решетки”, Вестн. Ивановского гос. ун-та, 2000, № 3, 116-122.

[9] Е.П. Барановский, "Жесткость L-разбиений решеток", Проблемы теоретической кибернетики, Тезисы XII Междунар. конф., М., 1999.

[10] M. Deza, V. Grishukhin, "Rank 1 forms, closed zones and laminae", J. Théor. Nombres Bordeaux, 14:1 (2002), 103-112.

[11] Б. А. Венков, "Об одном классе эвклидовых многогранников”, Вестн. ЛГУ, 1954, № 2, $11-31$.

[12] Е. П. Барановский, "Разбиение евклидовых пространств на L-многогранники некоторых совершенных решеток", Тр. МИАН, 196 (1991), 27-46.

[13] Дж. Конвей, Н. Слоэн, Упаковки шаров, решетки и группь, Мир, М., 1990.

[14] M. Deza, V. Grishukhin, "Delaunay polytopes of cut lattices", Linear Algebra Appl., 226228 (1995), 667-685.

\section{В. П. Гришухин}

Поступило

Центральный экономико-математический институт РАН

11.10 .2005

E-mail: grishuhn@cemi.rssi.ru

Исправленный вариант

10.01.2006 